\title{
Implementing a digital health model of care in Australian youth mental health services: protocol for impact evaluation
}

Sarah Piper*, Tracey A. Davenport, Haley LaMonica, Antonia Ottavio, Frank lorfino, Vanessa Wan Sze Cheng, Shane Cross, Grace Yeeun Lee, Elizabeth Scott and lan B. Hickie

\begin{abstract}
Background: The World Economic Forum has recently highlighted substantial problems in mental health service provision and called for the rapid deployment of smarter, digitally-enhanced health services as a means to facilitate effective care coordination and address issues of demand. In mental health, the biggest enabler of digital solutions is the implementation of an effective model of care that is facilitated by integrated health information technologies (HITs); the latter ensuring the solution is easily accessible, scalable and sustainable. The University of Sydney's Brain and Mind Centre (BMC) has developed an innovative digital health solution - delivered through the Youth Mental Health and Technology Program - which incorporates two components: 1) a highly personalised and measurement-based (data-driven) model of youth mental health care; and 2) an industrial grade HIT registered on the Australian Register of Therapeutic Goods. This paper describes a research protocol to evaluate the impact of implementing the BMC's digital health solution into youth mental health services (i.e. headspace - a highly accessible, youth-friendly integrated service that responds to the mental health, physical health, alcohol or other substance use, and vocational concerns of young people aged 12 to 25 years) within urban and regional areas of Australia.

Methods: The digital health solution will be implemented into participating headspace centres using a naturalistic research design. Quantitative and qualitative data will be collected from headspace health professionals, service managers and administrators, as well as from lead agency and local Primary Health Network (PHN) staff, via service audits, Implementation Officer logs, online surveys, and semi-structured interviews, at baseline and then threemonthly intervals over the course of 12 months.

Discussion: At the time of publication, six headspace centres had been recruited to this study and had commenced implementation and impact evaluation. The first results are expected to be submitted for publication in 2021. This study will focus on the impact of implementing a digital health solution at both a service and staff level, and will evaluate digital readiness of service and staff adoption; quality, usability and acceptability of the solution by staff; staff self-reported clinical competency; overall impact on headspace centres as well as their lead agencies and local PHNs; and social return on investment.
\end{abstract}

Keywords: Mental health, Digital health, Digital health solution, Health information technology, Mental health services, Young people, Implementation, eHealth

\footnotetext{
* Correspondence: sarah.piper@sydney.edu.au

The University of Sydney, Brain and Mind Centre, 94 Mallett St, Camperdown,

Sydney, NSW 2050, Australia
}

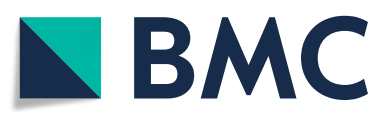

(c) The Author(s). 2021 Open Access This article is licensed under a Creative Commons Attribution 4.0 International License, which permits use, sharing, adaptation, distribution and reproduction in any medium or format, as long as you give appropriate credit to the original author(s) and the source, provide a link to the Creative Commons licence, and indicate if changes were made. The images or other third party material in this article are included in the article's Creative Commons licence, unless indicated otherwise in a credit line to the material. If material is not included in the article's Creative Commons licence and your intended use is not permitted by statutory regulation or exceeds the permitted use, you will need to obtain permission directly from the copyright holder. To view a copy of this licence, visit http://creativecommons.org/licenses/by/4.0/ The Creative Commons Public Domain Dedication waiver (http://creativecommons.org/publicdomain/zero/1.0/) applies to the data made available in this article, unless otherwise stated in a credit line to the data. 


\section{Background}

Young people aged 12 to 25 years have the highest incidence and prevalence of mental illness across the lifespan and bear a disproportionate share of the burden of disease associated with mental disorders [1-3]. Most recently, the Australian Child and Adolescent Survey of Mental Health and Wellbeing (conducted in 2013-14) estimates that 560,000 children and adolescents aged 4 to 17 years (almost 14\%) in Australia experienced a mental disorder in the 12 months before the survey [4]. Considering that $75 \%$ of serious mental illness, alcohol or other substance misuse occurs before the age of 25 years [5], early identification and intervention is crucial to prevent illness progression and reduce its impact. Left untreated, the ramifications of poor mental health can last a lifetime, often robbing the individual of their quality of life and costing the economy billions.

In response to this, the Australian Government established the National Youth Mental Health Foundation (headspace) in 2006 - with an aim to establish a highly accessible, youth-friendly, multi-disciplinary service that responds to the mental health, physical health, alcohol or other substance use, and vocational concerns of young people aged 12 to 25 years [6]. headspace aims to better integrate and coordinate the appropriate care for a young person, and ensure early detection and intervention of emerging mental and substance use disorders [6]. To achieve this, centres are staffed by multidisciplinary teams to address the multidimensional needs of young people, comprising of clinical staff (e.g. psychiatrists, mental health nurses, GPs; allied health professionals such a psychologists, occupational therapists, social workers), and non-clinical staff (e.g. centre manager, clinical coordinator, community outreach worker, intake workers, etc) [7]a. The composition and capacity of these teams vary across headspace centres nationally, due to factors such as geographical location (e.g. unavailable workforce in rural locations), funding arrangements, and the fact that the provision of treatment and services offered by a headspace centre is determined by the community in which it is located - i.e., headspace centres aim to reflect and serve the communities in which they are based. Despite the establishment of headspace, mental health outcomes for young people remain poor. For those who access care, a significant portion of young people receive an inappropriately low level of treatment for their needs [8], show no improvement in functioning over time [9], or deteriorate to a more serious stage of illness $[10,11]$.

Poor outcomes for young people with mental ill health can occur for a variety of reasons, many of which stem from the current mental health system's reliance on a 'traditional' model of service delivery (i.e. accessed in a 'bricks and mortar' service, utilising a waitlist, delivering exclusively face-to-face therapy, etc.) [12]. Within these traditional models of service delivery, not all Australians have access to high quality mental health care, and access to care varies greatly across geographical locations [13]. For example, people living in non-urban (regional, rural, remote) areas of Australia have limited access to mental health care [14] due to barriers such as location of service, service opening hours, the issue of anonymity (particularly in small rural or remote communities), stigmatising attitudes, and cost [15-17], and they experience higher self-harm, suicidal ideation, and suicide attempt rates than Australians living in urban areas [4]. Further, the quality and type of intervention received by a young person varies greatly across services and geographical locations [13]. There is limited communication and coordination between services, which leaves the young person navigating a disjointed mental health care system without support [18].

Recently, the World Economic Forum highlighted the substantial problems in mental health service provision and called for the rapid deployment of smarter, digitallyenhanced mental health services as a means to facilitate effective care coordination and address issues of demand [19]. A rapid increase in reported mental health issues during the COVID-19 pandemic has added to service demand, which cannot be met by current traditional service delivery models of mental health care [19]. Moreover, this surge in service demand has resulted in a significant increase in contacts with digital mental health services (e.g. Mindspot, Lifeline), further highlighting the important role digital health can play in providing mental health care on a large scale [20]. Yet, this increase also illustrates the need for better models of care otherwise we risk bringing more people into an already overburdened mental health system. Thus, the adoption of a digital health model of care by mental health services, and a shift in the historically cautious attitudes of clinicians toward the use of digital technologies [19], is now crucial in facilitating quality, timely, and easily accessible mental health care for all.

\section{Youth mental health and technology program}

The integration of health information technologies (HITs) into service delivery pathways is the biggest enabler of digital health [21-23], due to HITs being easily accessible, scalable, and sustainable. HITs have been defined as the application of information processing involving both computer hardware and software that deals with the storage, retrieval, sharing, and use of health care information, data and knowledge for communication and decision making' [24]. HITs can be utilised in a variety of health care settings (e.g. private and public sectors, primary, secondary and hospital services), and examples of HITs include electronic health records, 
medical billing software, and clinical decision support (a data-analysis system designed to support health professionals to make clinical decisions). Previous research has identified the key uses of HITs as being the storage, management, and transmission of health data; clinical decision making support; and the facilitation of health care from a distance [25]. HITs are currently transforming both the healthcare system and the ability of consumers to self-manage aspects of their health and wellbeing through exercising greater choice and control in their own care [26-30]. For mental health service providers and health professionals who employ shared decision-making, HITs are also transforming the ways in which consumers can choose to become active participants and equal partners in their health care, shifting service delivery from an intervention model to an effective coordinated care model that is person-centred and quality-driven [29-32]. The adoption of HITs in youth mental health services has already demonstrated their utility to improve access and triage to care, as well as communication between health professionals and consumers, particularly in regional, rural or remote settings [17].

Researchers at The University of Sydney's Brain and Mind Centre (BMC) have developed the Youth Mental Health (YMH) and Technology Program which utilises an innovative digital health model of care to increase access to quality mental health care for young people, improve mental, physical and social outcomes for young people, and upskill the youth mental health workforce to confidently deliver quality care. This digital health solution - delivered through the YMH and Technology Program - incorporates two components. The first is our highly personalised and measurement-based (datadriven) model of youth mental health care (known as the BMC Youth Model) [33], which has been developed from more than 10 years of clinical research from the BMC's Optymise Youth Cohort [11, 34]. The BMC Youth Model integrates a variety of clinical concepts, including a multidimensional assessment and outcomes framework (i.e. fostering holistic practice of mental health that considers other compounding comorbidities such as physical health), underlying pathophysiological mechanisms and illness trajectories (i.e. adopting a transdiagnostic framework that recognises that mental health conditions are rarely homogeneous), and clinical staging for mental health. Clinical staging models consider the spectrum of mental ill health and aim to place consumers on that continuum, from those with risk factors and symptoms or impairment (Stage 1a) or attenuated disorders (Stage 1b) through to those with discrete disorders or with persistent and recurrent syndromes (Stage 2+) [34]. Clinical stage separates young people based on differential risk of progression to more severe disorders and poorer outcomes, and is therefore an accurate and efficient guide to allocating care (i.e. a concept known as 'staged care') [35]. As a result of this research, the BMC Youth Model was subsequently translated into an education and training program [36], and its core concepts developed as key functionalities within an innovative HIT known as the InnoWell Platform [23, 37].

The second component of the digital health solution is a HIT, as exemplified by the InnoWell Platform [23, 37]. The InnoWell Platform is listed on the Australian Register of Therapeutic Goods (software as a medical device, class 1, ARTG ID 315030) as a customisable digital toolkit to assist assessment, monitoring, and management of mental ill health and maintenance of wellbeing. It does this by collecting, storing, scoring, and reporting personal and health information back to consumers and their health professionals to promote collaborative care partnerships [38]. The clinical content is determined by service providers. Importantly, the InnoWell Platform does not itself provide stand-alone medical or health advice, diagnosis, or treatment. Instead, it guides and supports, but does not direct, consumers and their treating health professionals to decide what may be suitable care options [38]. The InnoWell Platform is manufactured by InnoWell Pty Ltd. - a joint venture between the University of Sydney and PwC (Australia).

Therefore, our digital health solution facilitates use of the BMC Youth Model through the InnoWell Platform. Though we reference the InnoWell Platform as an exemplar HIT, it is important to note that the BMC Youth Model can be adopted via any HIT so long as its design has been guided by similar clinical and scientific concepts to provide highly personalised and measurementbased care.

This study aims to evaluate the impact of implementing the digital health solution (delivered through the YMH and Technology Program) into participating headspace centres within urban and regional areas of Australia. It focuses on impact at both a service and staff level, and will evaluate: digital readiness of service and staff adoption; quality, usability and acceptability of the solution; staff self-reported clinical competency; overall impact on headspace centres, their lead agencies and local Primary Health Networks (PHNs); as well as social return on investment.

\section{Methods}

\section{Study design and procedure}

\section{Implementation of the digital health solution}

The Implementation of the digital health solution into participating centres will be guided by our previously developed strategy for implementation science [39]. This strategy was developed and tested through previous 
research that implemented a HIT across a range of services and associated populations, including youth mental health (headspace), the veterans community (Open Arms - Veterans and Families Counselling), and eating disorder support services (Butterfly's National Helpline), as part of Project Synergy (a three year, Australian government -funded project which aimed to transform mental health services through the use of technology $[23,38])$.

The implementation strategy consists of four phases: Phase 1) scoping and feasibility (determine the fit regarding the aims of the service and the solution provided by the HIT); Phase 2) utilise co-design methodologies (including participatory design, service pathway modelling and user testing) to determine how the HIT can enhance the service; Phase 3) implement the HIT into the service, iteratively evaluating the solution over time to respond to the needs of the service (including staff, individuals, and individuals' support people); and Phase 4) the sustainment of the optimised HIT within the service (informed by phases 1 to 3 ), to achieve mental health service transformation through technology [39].

Implementation will follow a naturalistic research design, with each participating centre utilising the digital health solution for a period of 12 months. The naturalistic design will allow the digital health solution to be evaluated under ecologically valid conditions that reflect the customisable and changeable nature of the digital health solution in a 'real-world' youth mental health service setting. The order in which centres will implement the digital health solution will be naturalistically guided by each centre's unique circumstance, as per Phase one of our implementation strategy, 'scoping and feasibility'. Participating services will be offered the option of continuing to utilise the digital health solution as part of service delivery after the 12 month implementation period, without the accompanying impact evaluation research measures.

\section{Participating centres}

A minimum of six headspace centres across Australia will be offered the opportunity to implement the digital health solution into their service. Centres will be selected based on interest from the centres' commissioning body (their Primary Health Network [PHN]). The study's Coordinating Principal Investigator, supported by the study's co-investigators, will approach the headspace centre's relevant contact (e.g. service manager), via phone or email to offer further information regarding the implementation of the digital health solution. Centre managers will be contacted directly to ensure no selection bias with regards to PHN involvement. Centres will then commence phase one of the implementation science strategy (scoping and feasibility) [39].

\section{Impact evaluation}

Impact evaluation of the digital health solution has been guided and adapted by our previous work [40], which established data collection methods specifically to evaluate the impact of HITs for Australian mental health services reform. Methods include service audits, Implementation Officer logs, online surveys, and semistructured interviews, allowing us to collect longitudinal quantitative and qualitative data. This unique use of methodological triangulation (i.e. mixed methods) ensures that data collection will be comprehensive and inclusive of all stakeholders to drive enhanced understanding of the potential impacts of implementation. The triangulation of data has previously been used to evaluate the implementation of HITs amongst health service professionals [41, 42].

Service audits Service audits will collect nonidentifiable and aggregated data from participating headspace centres' relevant electronic health records software (e.g. Best Practice, Medical Director) to assess domains of clinical safety and service quality, such as accessibility and equity, acceptability and satisfaction, workforce competence and capability, and so on.

Implementation officer logs Implementation Officers, part of the Youth Mental Health and Technology research team at the University of Sydney's Brain and Mind Centre, will be embedded into participating headspace centres to support implementation. Implementation Officers will have a background working in the provision of mental health services (either clinical or non-clinical [e.g. administrative, research-focussed]), and will be embedded into participating centres part-time, contingent upon the varying needs and capacities of the services. Other tasks of Implementation Officers will include acting as a point of contact for participating sites, managing the recruitment of participants, overseeing informed consent, distributing online surveys to participants, administering semi-structured interviews, and routinely completing the Implementation Officer Log. The Implementation Officer log is based on the Quality Implementation Framework [43], and will be used to monitor changes in implementation, reimagined service pathways, and staff roles related to the digital health solution. Importantly, Implementation Officers are not making any observations about centre staff specifically (e.g. performance), but rather the processes of implementing the digital health solution within participating headspace centres. Implementation Officers will complete logs fortnightly through REDCap, a secure web application for building and managing online surveys and databases [44, 45]. Table 1 provides an example of the range of questions included in the Implementation 
Table 1 Example questions from the Implementation Officer $\log$

\begin{tabular}{|c|c|}
\hline $\begin{array}{l}\text { All questions are asked re } \\
\text { experience over the past } t\end{array}$ & $\begin{array}{l}\text { ling the Implementation Officer's } \\
\text { weeks. }\end{array}$ \\
\hline Category & Question \\
\hline Service-level impacts & $\begin{array}{l}\text { Is the digital health solution changing } \\
\text { and/or improving the following aspects } \\
\text { of the mental health service? If yes, how? } \\
\text { If no, why not? } \\
\text { i) Clinical safety } \\
\text { ii) Accessibility } \\
\text { iii) Continuity of care } \\
\text { iv) The delivery of staged care } \\
\text { v) Etc. }\end{array}$ \\
\hline Capacity/readiness & $\begin{array}{l}\text { Have there been any changes to the } \\
\text { service's capacity (e.g. resources, skills, } \\
\text { motivation)? What changes? }\end{array}$ \\
\hline $\begin{array}{l}\text { Quality and usability of the } \\
\text { digital health solution }\end{array}$ & $\begin{array}{l}\text { Does the platform require modifications } \\
\text { to improve its performance? Yes/No, } \\
\text { describe. } \\
\text { Does the Platform deliver adequate } \\
\text { functionality to support the BMC Youth } \\
\text { Model? Yes/no, describe. }\end{array}$ \\
\hline Implementation & $\begin{array}{l}\text { What aspects of the digital health } \\
\text { solution and its implementation have } \\
\text { been effective within the service? }\end{array}$ \\
\hline
\end{tabular}

Officer log, a full version of the log is provided in Additional file 1: Appendix A.

Online surveys and semi-structured interviews Quantitative and qualitative data will also be collected from participants via cross-sectional online surveys using REDcap [44, 45] and semi-structured interviews will be conducted on site at participating headspace centres. For participants who are unable to attend a headspace centre, or if in-person interviews are not appropriate (i.e. due to COVID-19 health restrictions), participants will be offered telephone or online video-conference interviews using Zoom, a secure cloud-based videoconferencing service with end-to-end chat encryption. Example questions from the online surveys and semistructured interviews are provided in Tables 2 and 3 respectively. The full version of the online survey and semi-structured interview are provided in Additional files 2 and 3: Appendices B and C, respectively.

Table 4 presents a summary of the impact evaluation outcome data collected (e.g. digital readiness and competence of staff, adoption of the digital health solution, etc), the method with which this data was collected (e.g. online survey, semi-structured interview), and the data collection timepoint.

\section{Participant recruitment} Impact evaluation

All staff within participating headspace centres that are using the digital health solution will be invited to
Table 2 Example questions from the online survey

Topic: Views on digital health, and the adoption of the digital health solution

Question Answer

In the last 2 weeks, to what extent did you employ the following in your usual clinical care:

What is the main reason for not always adopting any of the previous items in your usual clinical care? Please select all that apply.

(N/A not at all/ rarely/ sometimes/ very often/ always)

$\square$ Broad, multi-dimensional assessment of needs beyond mental health, including but not limited to: physical health, daily functioning, alcohol and drug use, and social connectedness

$\square$ Outcome monitoring to routinely measure a young person's progress using objective, standardised measures to track improvements or deterioration, for the purposes of treatment planning

$\square$ Match the 'intensity' of an intervention to the needs of the young person

$\square$ Shared or collaborative decision making with the young person under your care $\square$ Etc.

$\square$ I do not think any of the above items are important to adopt in my usual clinical care

口 I am worried that the digital health solution poses a potential risk to the quality of the care provided to clients $\square$ Time constraints $\square$ Capacity restraints $\square$ Etc.

When considering the positive social benefits you identified in the previous question, how much do you personally agree or disagree with the following statements?

(Not sure/ strongly disagree/ disagree/ neutral/ agree/ strongly agree)

$\square$ Without the Brain and Mind Centre's digital health solution, these positive social benefits (on consumers, my standard practice or my health service) would have happened anyway $\square$ Due to the Brain and Mind Centre's digital health solution, other tasks I used to carry out have stopped or have been replaced $\square$ These positive social benefits will continue in the years to come

participate in the impact evaluation study (including health professionals [psychologists, psychiatrists, social workers, occupational therapists, general practitioners, nurses, etc], service managers and administrators). Additionally, relevant staff from the headspace centre's lead agency and local PHN who were involved in the implementation of the digital health solution (e.g. project managers, project officers) will also be invited to participate.

Awareness of this study will be raised through the use of study advertisements (i.e. posters and postcards) displayed in staff areas, and through verbal conversations 
Table 3 Example questions used during a semi-structured interview

\begin{tabular}{|c|c|}
\hline \multicolumn{2}{|c|}{ Topic: Digital readiness and staff competence } \\
\hline Question & Answer \\
\hline $\begin{array}{l}\text { When it comes to the use of } \\
\text { digital health in your work, would } \\
\text { you say you are keeping up, or } \\
\text { falling behind? }\end{array}$ & $\begin{array}{l}\square \text { Keeping up } \\
\text { - What helps you to keep up (e.g. } \\
\text { personal interest, training provided } \\
\text { by service, etc)? } \\
\text { - What are the enablers to using } \\
\text { digital health solutions? } \\
\text { - When you describe yourself as } \\
\text { 'keeping up,' to whom are you } \\
\text { comparing yourself (e.g. colleagues, } \\
\text { etc.)? } \\
\square \text { Falling behind } \\
\text { - Why do you think you are falling } \\
\text { behind? } \\
\text { - Do you prefer not to use digital } \\
\text { health solutions in your work? } \\
\text { - Is your use of digital health } \\
\text { solutions at work different from } \\
\text { your colleagues? } \\
\text { - Etc. }\end{array}$ \\
\hline
\end{tabular}

with on-site Implementation Officers one month prior to scheduled research activity to allow potential participants time to consider participation. To avoid perceived coercion, interested participants will need to contact the Implementation Officer to receive a Participant Information Sheet, which will provide further information regarding the study, and state that there is no obligation for any staff member to take part in the study, and that choosing not to participate, or withdrawing from the study, will cause no detriment to their career or future employment. Participants will be asked to complete and sign the Participant Consent Form with options for them to consent to receiving links to the baseline and follow up online surveys and semi-structured interviews, as well as to consent to having their interview scribed and

\section{Reimbursement}

If participants are likely to experience any loss of earnings (e.g. contract health professionals), then this loss may be provided for by an honorarium, in line with the Medicare rebate they would have received from the Australian public health system.

\section{Data analysis}

Aggregate service-level outcome data will be compared across time points (baseline, three months, six months, nine months, 12 months), to identify change over time within services (specifically through calculating reliable change scores and effect sizes). As the aim of analyses is to determine whether the solution is effective for each individual service, sample size computation is not appropriate.

Data from online surveys will be analysed using descriptive statistics, as well as bivariate analyses using Fisher exact tests to evaluate group differences in each participating service. A reliability analysis will be carried out to evaluate internal consistency of the online surveys. All quantitative data will be analysed using the Statistical Package for the Social Sciences (SPSS) version 24 (IBM Corp).

Thematic analysis will be performed on qualitative data, including semi-structured interviews and implementation officer logs, to identify major themes relating to implementation of this digital solution across services, and will follow established thematic analysis techniques [46]. Interviews will be audio-recorded, transcribed and de-identified. Transcripts of both interviews and implementation officer logs will be coded using NVivo 12 software, and two independent knowledge translators from the research team will iteratively examine, discuss, and code the data, to establish a consensus regarding a

Table 4 Impact evaluation outcome data collection

\begin{tabular}{|c|c|c|c|c|}
\hline Data collection source & Online survey & Semi-structured interview & Implementation log & Service audit \\
\hline Collection time point & Three-monthly & Three-monthly & Monthly & Three-monthly \\
\hline \multirow[t]{6}{*}{$\begin{array}{l}\text { Impact evaluation } \\
\text { outcome collected }\end{array}$} & $\begin{array}{l}\text { Adoption of digital } \\
\text { health solution }\end{array}$ & Impact of digital health solution & Impact of digital health solution & Client safety \\
\hline & $\begin{array}{l}\text { Staff views on digital } \\
\text { health }\end{array}$ & $\begin{array}{l}\text { Quality, acceptability, usability of the } \\
\text { digital health solution }\end{array}$ & $\begin{array}{l}\text { Quality, acceptability, usability of the } \\
\text { digital health solution }\end{array}$ & $\begin{array}{l}\text { Client accessibility and } \\
\text { equity }\end{array}$ \\
\hline & $\begin{array}{l}\text { Education and training } \\
\text { outcomes }\end{array}$ & Education and training outcomes & Education and training outcomes & $\begin{array}{l}\text { Workforce (staff numbers, } \\
\text { FTE) }\end{array}$ \\
\hline & & Digital readiness and staff competence & Implementation barriers and facilitators & $\begin{array}{l}\text { Service efficiency, } \\
\text { expenditure, and cost }\end{array}$ \\
\hline & & & & $\begin{array}{l}\text { Service effectiveness and } \\
\text { outcomes }\end{array}$ \\
\hline & & & & $\begin{array}{l}\text { Service continuity and } \\
\text { coordination }\end{array}$ \\
\hline
\end{tabular}

Note: data collected at three-monthly timepoints (baseline, three-months, six-months, nine-months, and 12-months) will cover the preceding three months. Data collected at monthly timepoints will cover the preceding month

audio-recorded.

coding framework. 


\section{Data storage and security}

For the duration of the research, all electronic data from service audits, Implementation Officer logs, online surveys and semi-structured interviews will be fed into a secure password-protected virtual machine within the Research Data Store provided by The University of Sydney. All service audit, Implementation Officer log, and online survey data will be non-identifiable, and semistructured interviews will be de-identified if required. Upon completion of this research, all electronic data will be stored on a secured password-protected virtual machine within the Research Data Store provided by The University of Sydney.

\section{Ethics and dissemination}

Ethics approval has been granted via Sydney Local Health District's Human Research Ethics Committee (HREC) (Protocol No X18-0499 \& HREC/18/RPAH/ 715 ), and via The University of Sydney HREC (Project No 2018/849). Site-specific approvals were obtained when required.

\section{Discussion}

At the time of publication, six headspace centres had been recruited to this study and had commenced implementation and impact evaluation. The first results are expected to be submitted for publication in 2021.

Nationally, youth mental health is a priority as most mental disorders emerge before a young person turns 25 years old; and if not treated early enough, these disorders become lifelong impairments. Given recent world events such as the COVID-19 pandemic, there has never been a more critical time to focus on improving the mental health and wellbeing of young Australians. As a consequence, digital health models of care are now seen as crucial solutions for youth mental health services to rapidly increase access to quality mental health care [47].

The aim of this study is to describe a research protocol to evaluate the impact of implementing a digital health solution (delivered via the YMH and Technology Program) into headspace centres within urban and regional areas of Australia. It focuses on the impact of the digital health solution on staff and service, to better understand the importance of digital readiness, clinical competency and confidence, and the quality, usability, acceptability and overall impact of the digital health solution. The focus on staff and service-level data collection is integral to effective implementation of this digital health solution, and will provide critical feedback regarding the fit of the solution and identify service needs to ensure future successful implementations.

A consideration regarding future implementations is an evaluation of the effectiveness of an associated education and training program [36] that aims to upskill health professionals in the application of the digital health solution. Considering that clinical decisions made by health professionals are predominantly influenced by their knowledge, skills, and beliefs [13], the education and training of health professionals is central to adopting, sustaining and optimising the digital health solution in standard clinical practice.

\begin{abstract}
Abbreviations
BMC: Brain and Mind Centre; BMC Youth Model: Brain and Mind Centre Youth Model; HIT: Health Information Technology; PHN: Primary Health Network; YMH and Technology Program: Youth Mental Health and Technology Program; HREC: Human Research Ethics Committee; FGG: Future Generations Global
\end{abstract}

\section{Supplementary Information}

The online version contains supplementary material available at https://doi. org/10.1186/s12913-021-06394-4.

Additional file 1:. Implementation Officer Log

Additional file 2:. Baseline Online Survey

Additional file 3:. Baseline Interview Questions

Acknowledgements

The authors would like to acknowledge Future Generation Global for funding the Youth Mental Health and Technology Program, which aims to improve young people's access to quality mental health care.

Authors' contributions

$S P, H L, T A D, W W S C$, and GYL wrote and revised the manuscript. $A O, H L, F l$, $T A D, S C, E S$ and $I H$ significantly contributed to the development of the protocol. All authors read, revised, and approved the final manuscript.

\section{Funding}

This study was funded by Future Generation Global, who otherwise had no involvement in study design, collection, analysis, and interpretation of data, and manuscript preparation.

\section{Availability of data and materials}

The dataset of this study will be made available upon reasonable request of the corresponding author.

\section{Declarations}

Ethics approval and consent to participate

All trial sites included in this study have received ethics approval. Ethics approval has been granted via Sydney Local Health District's Human Research Ethics Committee (HREC) (Protocol No X18-0499 \& HREC/18/RPAH/ 715) for the following trial sites: headspace Ashfield, Bondi Junction, Hurstville, Miranda, Camperdown, and Edinburgh North.

As stated above under 'Participant recruitment', interested participants will need to contact the Implementation Officer (via email or in person on-site) to receive a Participant Information Sheet, and the Implementation Officer will then answer any outstanding questions regarding participation. If they would like to participate in the study, the potential participant will be provided with a written Participant Consent Form (provided in-person, on-site; or via email) which includes options for them to consent to receiving links to the baseline and follow up online surveys and semi-structured interviews, as well as to consent to having their interview scribed and audio-recorded. For semi-structured interviews, informed consent will be confirmed by the research team prior to the beginning of the session on the day. 


\section{Competing interests}

IBH was an inaugural Commissioner on Australia's National Mental Health Commission (2012-18). He is the Co-Director, Health and Policy at The University of Sydney's Brain and Mind Centre (BMC). The BMC operates an earlyintervention youth services at Camperdown under contract to headspace. IBH is the Chief Scientific Advisor to, and a 5\% equity shareholder in, InnoWell Pty Ltd. InnoWell was formed by the University of Sydney (45\% equity) and PWC (Australia; 45\% equity) to deliver the $\$ 30$ million Australian Government-funded Project Synergy (2017-20; a three-year program for the transformation of mental health services) and to lead transformation of mental health services internationally through the use of innovative technologies. Ms. Tracey Davenport is now the Director (Research and Evaluation), Design and Strategy Division, at the Australian Digital Health Agency. Other authors on this paper have no conflicts of interest to disclose.

\section{Received: 25 August 2020 Accepted: 14 April 2021}

Published online: 12 May 2021

\section{References}

1. Kessler RC, Berglund P, Demler O, Jin R, Merikangas KR, Walters EE. Lifetime prevalence and age-of-onset distributions of DSM-IV disorders in the National Comorbidity Survey Replication. Arch Gen Psychiatry. 2005;62(6): 593-602. https://doi.org/10.1001/archpsyc.62.6.593.

2. Merikangas KR, He JP, Burstein M, Swanson SA, Avenevoli S, Cui L, et al. Lifetime prevalence of mental disorders in U.S. adolescents: results from the National Comorbidity Survey Replication--Adolescent Supplement (NCS-A). J Am Acad Child Adolesc Psychiatry. 2010;49(10):980-9. https://doi.org/10.101 6/j.jaac.2010.05.017.

3. Slade T, Johnston A, Oakley Browne MA, Andrews G, Whiteford H. 2007 National Survey of mental health and wellbeing: methods and key findings. Aust New Zealand J Psychiatr. 2009:43(7):594-605. https://doi.org/10.1080/ 00048670902970882

4. Lawrence D, Johnson S, Hafekost J, Boterhoven de Haan K, Sawyer M, Ainley J, et al. The Mental Health of Children and Adolescents: Report on the second Australian Child and Adolescent Survey of Mental Health and Wellbeing. 2015.

5. Gore FM, Bloem PJN, Patton GC, Ferguson J, Joseph V, Coffey C, et al. Global burden of disease in young people aged 10\&\#x2013;24 years: a systematic analysis. Lancet. 2011;377(9783):2093-102. https://doi.org/10.101 6/S0140-6736(11)60512-6

6. McGorry PD, Tanti C, Stokes R, Hickie IB, Carnell K, Littlefield LK, et al. Headspace: Australia's National Youth Mental Health Foundation--where young minds come first. Med J Aust. 2007;187(S7):S68-70. https://doi.org/10 5694/j.1326-5377.2007.tb01342.x.

7. Rickwood D, Paraskasis M, Quin D, Hobbs N, Ryall V, Trethowan J, et al. Australia's innovation in youth mental health care: the headspace Centre model. Early Interv Psychiatr. 2019;13(1):159-66. https://doi.org/10.1111/ eip.12740.

8. Cross SPM, Hickie IB. Transdiagnostic stepped care in mental health. Public Health Res Pract. 2017;27(2):e2721712. https://doi.org/10.17061/phrp2721 712 .

9. Carpenter JS, lorfino F, Cross S, Nichles A, Zmicerevska N, Crouse JJ, et al. Cohort profile: the brain and mind Centre Optymise cohort: tracking multidimensional outcomes in young people presenting for mental healthcare. BMJ Open. 2020;10(3):e030985. https://doi.org/10.1136/ bmjopen-2019-030985

10. Cross SPM, Scott J, Hickie IB. Predicting early transition from sub-syndromal presentations to major mental disorders. BJPsych Open. 2017;3(5):223-7. https://doi.org/10.1192/bjpo.bp.117.004721.

11. Iorfino F, Scott EM, Carpenter JS, Cross SP, Hermens DF, Killedar M, et al. Clinical stage transitions in persons aged 12 to 25 years presenting to early intervention mental health services with anxiety, mood, and psychotic disorders. JAMA Psychiatr. 2019;76(11):1167-75. https://doi.org/10.1001/jama psychiatry.2019.2360

12. NSW Department of Health. Mental Health Clinical Care and Prevention Model: A population mental health model. Sydney: NSW Department of Health; 2001.

13. Buchan HA, Duggan A, Hargreaves J, Scott IA, Slawomirski L. Health care variation: time to act. Med J Aust. 2016;205(S10):S30-3.

14. National Mental Health Commission. Australian Mental Health Commissions - Submission to the accessibility and quality of mental health services in rural and remote Australia Senate Inquiry. https://nswmentalhea Ithcommission.com.au/sites/default/files/documents/mental_health_ commissions_submission_-_mental_health_services_in_rural_and_remote_a ustralia.pdf; 2018.

15. Gulliver A, Griffiths KM, Christensen H. Perceived barriers and facilitators to mental health help-seeking in young people: a systematic review. BMC Psychiatry. 2010;10(1):113. https://doi.org/10.1186/1471-244X-10-113.

16. Orygen. Inquiry into the accessibility and quality of mental health services in rural and remote Australia. 2018.

17. Rowe SC, Davenport TA, Easton MA, Jackson TA, Melsness J, Ottavio A, et al. Co-designing the InnoWell platform to deliver the right mental health care first time to regional youth. Aust J Rural Health. 2020;28(2):190-4. https:// doi.org/10.1111/ajr.12617.

18. National Mental Health Commission. The National Review of Mental Health Programmes and Services. https://www.mentalhealthcommission.gov.au/ Monitoring-and-Reporting/national-reports/2014-Contributing-Lives-Review: Sydney: National Mental Health Commission; 2014

19. World Economic Forum. How technology can help to manage COVID-19's mental health fallout. https://www.weforum.org/agenda/2020/08/covid-19mental-health-telehealth/; 2020

20. Titov N, Staples L, Kayrouz R, Cross S, Karin E, Ryan K, et al. Rapid report: early demand, profiles and concerns of mental health users during the coronavirus (COVID-19) pandemic. Internet Interv. 2020;21:100327. https:// doi.org/10.1016/j.invent.2020.100327.

21. Lora A, Lesage A, Pathare S, Levav I. Information for mental health systems: an instrument for policy-making and system service quality. Epidemiol Psychiatr Sci. 2017;26(4):383-94. https://doi.org/10.1017/S2045796016000743.

22. Kilbourne AM, Beck K, Spaeth-Rublee B, Ramanuj P, O'Brien RW, Tomoyasu $N$, et al. Measuring and improving the quality of mental health care: a global perspective. World Psychiatry. 2018;17(1):30-8. https://doi.org/10. 002/wps.20482

23. Hickie IB, Davenport TA, Burns JM. Project synergy: co-designing technology-enabled solutions for Australian mental health services reform Med J Aust. 2019;211(S7):S3-S39.

24. Brailer $\mathrm{D}$. The decade of health information technology: delivering consimer-centric and information-rich health care. Framework for Strategic Action 2004. Available from https://www.providersedge.com/ehdocs/ehr_a rticles/the decade of hit-delivering_customer-centric and info-rich_hc.pdf

25. Black A, Car J, Pagliari C, Anandan C, Crewsswell K, Bokun T, et al. The impact of ehealth on the quality and safety of health care: a systematic overview. PLoS Med. 2011;18(8):e1000387.

26. Lupton D. Self-tracking, health and medicine. Health Sociol Rev. 2017;26(1): 1-5. https://doi.org/10.1080/14461242.2016.1228149.

27. Hickson R, Talbert J, Thornbury WC, Perin NR, Goodin AJ. Online medical care: the current state of "eVisits" in acute primary care delivery. Telemed J E-health. 2015;21(2):90-6. https://doi.org/10.1089/tmj.2014.0022.

28. Hollis C, Morriss R, Martin J, Amani S, Cotton R, Denis M, et al. Technological innovations in mental healthcare: harnessing the digital revolution. $\mathrm{Br} \mathrm{J}$ Psychiatry. 2015:206(4):263-5. https://doi.org/10.1192/bjp.bp.113.142612.

29. Roberts LW, Kim JP. Are individuals living with mental illness and their preferred alternative decision-makers attuned and aligned in their attitudes regarding treatment decisions? J Psychiatr Res. 2016;78:42-7. https://doi. org/10.1016/j.jpsychires.2016.03.004

30. Castle-Clarke S, Imison C. The digital patient: transforming primary care? https://www.nuffieldtrust.org.uk/research/the-digital-patient-transformingprimary-care: Nuffield Trust; 2016

31. Falconer $\mathrm{E}$, Kho D, Docherty JP. Use of technology for care coordination initiatives for patients with mental health issues: a systematic literature review. Neuropsychiatr Dis Treat. 2018;14:2337-49. https://doi.org/10.2147/ NDT.S172810.

32. Harvey MJ, Harvey MG. Privacy and security issues for mobile health platforms. J Assoc Inf Sci Technol. 2014;65(7):1305-18. https://doi.org/10.1 002/asi.23066

33. Hickie IB, Scott EM, Cross SP, Iorfino F, Davenport TA, Guastella AJ, et al. Right care, first time: a highly personalised and measurement-based care model to manage youth mental health. Med J Aust. 2019;211(S9):S3-S46.

34. Iorfino F, Hermens DF, Cross SP, Zmicerevska N, Nichles A, Badcock CA, et al. Delineating the trajectories of social and occupational functioning of young people attending early intervention mental health services in Australia: a longitudinal study. BMJ Open. 2018:8(3):e020678. https://doi. org/10.1136/bmjopen-2017-020678. 
35. Hickie IB, Scott J, McGorry PD. Clinical staging for mental disorders: a new development in diagnostic practice in mental health. Med J Aust. 2013; 198(9):461-2. https://doi.org/10.5694/mja13.10431.

36. Dohnt H, Dowling M, Davenport TA, Lee G, Piper S, Scott E, et al. Supporting clinicians to deliver 21st-century youth mental health care: protocol for an evaluation study. (in submission). 2020.

37. Iorfino F, Cross SP, Davenport T, Carpenter JS, Scott E, Shiran S, et al. A digital platform designed for youth mental health services to deliver personalized and measurement-based care. Front Psychiatr. 2019;10:595. https://doi.org/10.3389/fpsyt.2019.00595.

38. Davenport TA, LaMonica HM, Whittle L, English A, lorfino F, Cross S, et al. Validation of the InnoWell platform: protocol for a clinical trial. JMIR Res Protocols. 2019;8(5):e13955. https://doi.org/10.2196/13955.

39. LaMonica HM, Davenport TA, Braunstein K, Ottavio A, Piper S, Martin C, et al. Technology-enabled person-centred mental health services reform: strategy for implementation science. JMIR Ment Health. 2019;6(8):e14719. https://doi.org/10.2196/14719.

40. LaMonica HM, Braunstein K, Rowe SC, Ottavio A, Jackson T, Easton MA, et al. Technology-enabled solutions for Australian mental health services reform: impact evaluation. JMIR Form Res. 2020;4(11):e18759. https://doi.org/10.21 96/18759.

41. Lennon MR, Bouamrane M-M, Devlin AM, O'Connor S, O'Donnell C, Chetty $\mathrm{U}$, et al. Readiness for delivering digital health at scale: lessons from a longitudinal qualitative evaluation of a National Digital Health Innovation Program in the United Kingdom. J Med Internet Res. 2017;19(2):e42. https:// doi.org/10.2196/jmir.6900.

42. Mair FS, May C, Finch T, Murray E, Anderson G, Sullivan F, et al. Understanding the implementation and integration of e-health services. J Telemed Telecare. 2007;13(1_suppl):36-7.

43. Meyers DC, Durlak JA, Wandersman A. The quality implementation framework: a synthesis of critical steps in the implementation process. Am J Community Psychol. 2012;50(3-4):462-80. https://doi.org/10.1007/s10464012-9522-X.

44. Harris PA, Taylor R, Thielke R, Payne J, Gonzalez N, Conde JG. Research electronic data capture (REDCap) - a metadata-driven methodology and workflow process for providing translational research informatics support. Biomed Inform. 2009:42(2):377-81. https://doi.org/10.1016/j.jbi.2008.08.010.

45. Harris PA, Taylor R, Minor BL, Elliott V, Fernandez M, O'Neal L, et al. The REDCap consortium: Building an international community of software platform partners. J Biomed Inform. 2019;95:103208.

46. Braun V, Clarke V. Using thematic analysis in psychology. Qual Res Psychol. 2006;3(2):77-101. https://doi.org/10.1191/1478088706qp063oa.

47. Hickie IB. The role of new technologies in monitoring the evolution of psychopathology and providing measurement-based care in young people. World Psychiatry. 2020;19(1):38-9. https://doi.org/10.1002/wps.20697.

\section{Publisher's Note}

Springer Nature remains neutral with regard to jurisdictional claims in published maps and institutional affiliations.

Ready to submit your research? Choose BMC and benefit from:

- fast, convenient online submission

- thorough peer review by experienced researchers in your field

- rapid publication on acceptance

- support for research data, including large and complex data types

- gold Open Access which fosters wider collaboration and increased citations

- maximum visibility for your research: over $100 \mathrm{M}$ website views per year

At $\mathrm{BMC}$, research is always in progress.

Learn more biomedcentral.com/submissions 